Nota científica

(Short communication)

\title{
PRIMER REGISTRO DE EUPELMUS CUSHMANI (CRAWFORD) (HYMENOPTERA: EUPELMIDAE) COMO PARASITOIDE DEL BARRENADOR DE RAMAS DEL AGUACATE COPTURUS AGUACATAE KISSINGER (COLEOPTERA: CURCULIONIDAE) EN MÉXICO
}

\author{
FIRST RECORD OF EUPELMUS CUSHMANI (CRAWFORD) (HYMENOPTERA: \\ EUPELMIDAE) AS PARASITOID OF THE AVOCADO STEM WEEVIL COPTURUS \\ AGUACATAE KISSINGER (COLEOPTERA: CURCULIONIDAE) IN MEXICO
}

\section{MARIO ORLANDO ESTRADA VIRGEN ${ }^{2}$, NDAHITA DE DIOS ÁVILA ${ }^{1}$, ENRIQUE RUÍZ CANCINO $^{3}$, JUANA MARÍA CORONADO BLANCO ${ }^{3}$, CLAUDIO RIOS VELASCO ${ }^{4}$, OCTAVIO JHONATHAN CAMBERO CAMPOS $^{1,2 *}$}

\footnotetext{
${ }^{1}$ Posgrado en Ciencias Biológico Agropecuarias. Universidad Autónoma de Nayarit. Carretera Tepic-Compostela Km. 9. Xalisco, Nayarit, México. <ndahitadedios@gmail.com>

${ }^{2}$ Unidad Académica de Agricultura, Universidad Autónoma de Nayarit, Carretera Tepic-Compostela Km. 9. C.P. 63155. Xalisco, Nayarit, México. <estra0288@gmail.com>; <jhony695@gmail.com>

${ }^{3}$ Facultad de Ingeniería y Ciencias, Universidad Autónoma de Tamaulipas, Centro Universitario Adolfo López Mateos, Cd. Victoria, Tamaulipas, México, C.P. 87149.<eruiz@uat.edu.mx>; <jmcoronado@uat.edu.mx>

${ }^{4}$ Centro de Investigación en Alimentación y Desarrollo A. C., Unidad Cuauhtémoc, Av. Río Conchos S/N Parque Industrial. C.P. 31570, Chihuahua, México.<claudio.rios@ciad.mx>

*Autor de correspondencia: <jhony695@gmail.com>
}

Recibido: 22/09/2016; aceptado: 11/10/2018; publicado en línea: 29/03/2019 Editor responsable: Pedro Reyes Castillo†; Sergio Ibáñez-Bernal

Estrada-Virgen, M. O., De Dios, A. N., Ruíz-Cancino, E., Coronado-Blanco, J. M., Rios-Velasco, C., Cambero-Campos, O. J. (2019) Primer registro de Eupelmus cushmani (Crawford) (Hymenoptera: Eupelmidae) como parasitoide del barrenador de ramas del aguacate Copturus aguacatae Kissinger (Coleoptera: Curculionidae) en México. Acta Zoológica Mexicana (nueva serie), 35, 1-3. https://doi.org/10.21829/azm.2019.3502194

RESUMEN. Se registra por primera vez a Eupelmus cushmani (Crawford) (Hymenoptera: Eupelmidae) como parasitoide del barrenador de ramas del aguacate, Copturus aguacatae Kissinger en Nayarit, México.

Palabras clave: Barrenador; aguacate; control biológico; Nayarit

Estrada-Virgen, M. O., De Dios, A. N., Ruíz-Cancino, E., Coronado-Blanco, J. M., Rios-Velasco, C., Cambero-Campos, O. J. (2019) First record of Eupelmus cushmani (Crawford) (Hymenoptera: Eupelmidae) as parasitoid of the avocado stem weevil Copturus aguacatae Kissinger (Coleoptera: Curculionidae) in Mexico. Acta Zoológica Mexicana (nueva serie), 35, 1-3. https://doi.org/10.21829/azm.2019.3502194

ABSTRACT. Eupelmus cushmani (Crawford) (Hymenoptera: Eupelmidae) is recorded for first time as parasitoid of the avocado stem weevil (Copturus aguacatae Kissinger) in Nayarit, Mexico. 
Key words: Stem weevil; avocado; biological control; Nayarit

En México, el aguacate (Persea americana Mill.) es un cultivo comercialmente rentable donde la mayor parte de la producción se exporta a Estados Unidos de América y Japón (SAGARPA, 2014), sin embargo, su producción es limitada por diversos problemas fitosanitarios como es el caso del barrenador de ramas Copturus aguacate Kissinger (Equihua et al., 2007). Debido a sus hábitos alimentarios (Barrenador), causan severos daños en todos los estados fenológicos de la planta. El control de esta plaga se basa principalmente por el uso exclusivo de productos químicos, el abuso indiscriminado ha traído resultados poco efectivos y problemas de resistencia (APEAM, 2016). Lo anterior abre la posibilidad de emplear otras opciones de manejo de esta plaga, como el uso de enemigos naturales.

En México la familia Eupelmidae cuenta con ocho géneros y 21 especies (Noyes, 2016). La mayoría de las especies de Eupelmidae se caracteriza por parasitar larvas o prepupas de insectos por lo general ocultos en plantas o capullos (Gibson et al., 1997).

Eupelmus cushmani es considerado como parasitoide de 77 especies en 21 familias de cinco órdenes de insectos; en donde destaca el orden Coleoptera con el mayor número de especies hospederas. Se encuentra ampliamente distribuido, en las regiones Neártica y Neotropical (Noyes, 2016). En México Pérez y Bonet (1985) registraron por primera vez en Tepotzotlán, Morelos, la presencia de E. cushmani parasitando Acanthoscelides obtectus Say (Coleoptera: Bruchidae) mientras que Rodríguez et al. (2012) registraron al picudo del chile Anthonomus eugenii Cano (Coleoptera: Curculionidae) como nuevo hospedero de E. cushmani en Nayarit, México. En el presente trabajo se registra por primera vez a Eupelmus cushmani (Crawford) como parasitoide de Copturus aguacatae Kissinger en el estado de Nayarit, México.

Se recolectaron ramas de aguacate (material biológico) con sintomatología característica de daño por $C$. aguactae durante febrero a diciembre del 2015, en huertos de aguacate cultivar Hass en los municipios de Tepic y Xalisco del estado de Nayarit, México. Se realizaron muestreos con base en la metodología descrita en la NOM-066-FITO-2002 (SAGARPA, 2005), la cual consiste en seleccionar diez árboles de aguacate/ha al azar, inspeccionándose visualmente cuatro ramas de cada árbol, una en cada punto cardinal. El material biológico se trasladó al Laboratorio de Parasitología Agrícola del Centro Multidisciplinario de Investigación Científica (CEMIC) de la Universidad Autónoma de Nayarit (UAN), donde se criaron las ramas en vasos de platico de 1 L (Reyma ${ }^{\circledR}$ ), con una perforación en la tapadera y sellado con tela de organza, con el fin de mantener los pedazos de ramas en condiciones adecuadas para que los insectos completaran su desarrollo. Se incubaron a una temperatura de $25 \pm 2^{\circ} \mathrm{C}$ con un fotoperiodo de 12:12. Se realizaron revisiones diarias para registrar los parasitoides emergidos; los insectos obtenidos se etiquetaron y preservaron en alcohol al 70\%, para su posterior identificación usando las claves taxonómicas de Gibson et al. (1997) y Gibson (2011).

Material examinado. Eupelmus cushmani. México: Nayarit, Tepic predio Camichin I: 27-III-2015 (1

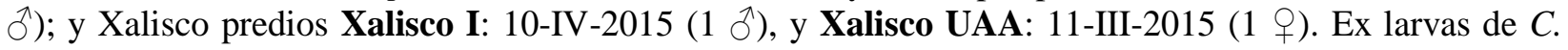
aguacatae. Ndahita de Dios Avila (Col.). El material se encuentra depositado en la colección del Laboratorio de Parasitología Agrícola del Centro Multidisciplinario de Investigación Científica (CEMIC) de la Universidad Autónoma de Nayarit (UAN) en Tepic, Nayarit, México.

Agradecimientos. Al Posgrado en Ciencias Biológico Agropecuarias de la Universidad Autónoma de Nayarit y al Consejo Nacional de Ciencia y Tecnología (CONACyT) por el apoyo económico brindado. 


\section{LITERATURA CITADA}

APEAM (2016) Asociación de Productores y Empacadores Exportadores de Aguacate de México, A. C. Listado de plaguicidas recomendados para el cultivo de aguacate. Disponible en: http://plaguicidas.apeamac.com/ArchivoPDF.aspx (acceso 14 de abril de 2016).

Equihua-Martínez, A. Estrada-Venegas, E. G., González-Hernández, H. (2007) Plagas, pp: 133-165. In: Téliz, D., Mora, A. (Eds.). El aguacate y su manejo integrado. Ediciones Mundi Prensa, México, D.F.

Gibson, G. A. P. (2011) The species of Eupelmus (Eupelmus) Dalman and Eupelmus (Episolindelia) Girault (Hymenoptera: Eupelmidae) in North America north of Mexico. Zootaxa, 2951, 33-41.

Gibson, G. A. P., Humber, J. T., Woolley, J. B. (1997) Annotated keys to the genera of Nearctic Chalcidoidea (Hymenoptera). NCR Research Press, Ottawa, Ontario, Canadá, 431 pp.

Noyes, J. S. (2016) Universal Database Chalcidoidea. Disponible en: http://www.nhm.ac.uk/ourscience/data/chalcidoids/database/chalcidsByCountry.dsml?index=ChalcidsByCountry\&COUNT $\mathrm{RY}=\mathrm{MEX} \&$ beginIndex $=700 \&$ searchPageURL=indexChalcidsByCountry $\% 2$ edsml $\% 3$ findex $\% 3 \mathrm{~d}$ ChalcidsByCountry\%26COUNTRY\%3dMEX\%26beginIndex\%3d600 (acceso 12 de junio de 2016).

Peréz, G., Bonet, A. (1985) Hymenopterous parasites of Acanthoscelides obtectus (Say) (Coleoptera: Bruchidae) in Tepoztlan, Morelos. Folia Entomológica Mexicana, 59, 71-78.

Rodríguez, L. E., Lomeli F. J., Valdez, C. J., Jones R. W., Stansly P. A. (2012) New records of species and locations of parasitoids of the pepper weevil in Mexico. Southwestern Entomologist, 37, 7383.

SAGARPA (2005) (Secretaria de Agricultura, Ganadería, Desarrollo Rural, Pesca y Alimentación) Norma Oficial Mexicana Fitosanitaria NOM-066-FITO-2002, Especificaciones para el manejo fitosanitario y movilización del aguacate. Diario oficial. Fecha de publicación 18 de mayo de 2005. $45 \mathrm{pp}$.

SAGARPA (2014) (Secretaria de Agricultura, Ganadería, Desarrollo Rural, Pesca y Alimentación) Comunicado de prensa. Disponible en http://www.sagarpa.gob.mx/saladeprensa/2012/Paginas /2014B615.aspx (acceso 15 de junio de 2016). 